\title{
Design and Investigation of a Reusable Surface-Mounted Optical Fiber Bragg Grating Strain Sensor
}

\author{
Yongxing Guo, Member, IEEE, Jianyi Kong, Honghai Liu, Senior Member, IEEE, Dongtao Hu, and Li Qin
}

\begin{abstract}
This paper describes the structure design, parameters optimization, and performance test of a fiber Bragg grating strain sensor with features of surface-mounting and reusability. Flexure hinges are adopted in the design of a key elastic body. Number of mounting holes, sealing, sensitization, and stiffness of the sensor have been considered and optimized. Then, sensor prototype has been manufactured and fully tested. The experimental results show that the stiffness and the sensitizing coefficient are identical to design the values. Test results also show that the sensor provides a sensitivity of $3.357 \mathrm{pm} / \mu \varepsilon$ with a fitting linear correlation coefficient of 0.9999 , a measurement range of $\pm 600 \mu \varepsilon$, a repeatability error of $2.73 \%$, and a hysteresis error of $4.67 \%$. Furthermore, good creep resistance and good capability for alternating strain measurement have also been demonstrated. These results promise the sensor potential applications in structural health monitoring.
\end{abstract}

Index Terms-Fiber Bragg grating (FBG), strain sensor, reusable, structural health monitoring.

\section{INTRODUCTION}

S TRAIN measurement is a basic and important subject in mechanical equipment and civil engineering. Accurate and effective strain measuring instrument can provide the deformation characteristics and trends of the measured object, which are important references for engineers to analyze deformation behavior and optimization design. The traditional strain measuring methods are mostly based on resistance strain gauge or vibration wire [1], [2], however, these electromagnetic sensing elements output weak voltage signal which is vulnerable to outside magnetic interference, tedious wire jointing, zero temperature drift, and rapid signal attenuation with long wire.

Manuscript received July 23, 2016; revised August 31, 2016; accepted September 25, 2016. Date of publication September 27, 2016; date of current version November 4, 2016. This work was supported in part by the National Natural Science Foundation of China under Grant 51605348 and Grant 51509194, in part by the China Postdoctoral Science Foundation under Grant 2015M572208, and in part by the Key Laboratory of Metallurgical Equipment and Control of Education Ministry, Wuhan University of Science and Technology Foundation under Grant 2015B04. The associate editor coordinating the review of this paper and approving it for publication was Dr. Minghong. Yang.

Y. Guo, J. Kong, and L. Qin are with the School of Machinery and Automation, Wuhan University of Science and Technology, Wuhan 430081, China (e-mail: yongxing_guo@wust.edu.cn; kongjianyi@wust.edu.cn; qinli429@wust.edu.cn).

H. Liu is with the Intelligent Systems and Biomedical Robotics Group, University of Portsmouth, Portsmouth, PO1 3HE, U.K. (e-mail: honghai. liu@port.ac.uk).

D. $\mathrm{Hu}$ is with the School of Resources and Environmental Engineering, Wuhan University of Technology, Wuhan 430070, China (e-mail: eastao@163.com).
These drawbacks restrict their practical application for long term and long distance monitoring, especially in wild and harsh environment [3], [4].

Recent years, optical fiber sensors offer a promising alternative to electric measuring elements. As one of the most widely used fiber sensing element, the FBG (Fiber Bragg grating) has been proven to be very effective because of its distinguishing advantages, such as immunity to electromagnetic interference and optical power fluctuations, high resistance to hostile environments, no zero temperature drift and the fact that multiple FBGs can be arrayed along a single optical fiber [5]. In the past two decades, FBG sensors have been widely used in structural health monitoring including mechanical equipment [6], aircraft [7], slope [8], [9], dam [10], etc, and other areas such as humidity [11] and biomedicine [12]. In these applications, strain measurement is essential and the most widely needed.

According to the method of installation, FBG based strain measurement can be classified into two types: surface-mounted strain sensor and embedded-mounted strain sensor. Embeddedmounted FBG strain sensors need to be imbedded into the body of measured object during building or manufacturing process of the object, for example, composite material [13] and armored concrete [14]. Bare FBGs are usually embedded in composite materials, whereas well-packaged FBG strain sensors in armored concrete. The well-packaged embeddable strain sensors are widely used today in concrete structures such as bridge, tunnel, dam, and building. The structure is usually a cylinder with two disk ends in which the FBG is packaged [15], [16]. However, because the demand of being embedded when the measured object is constructed, the embedded-mounted FBG sensors can't be replaced once they are failed during the long-term monitoring.

Actually, many structure projects have not been considered to carry out health monitoring when constructed until danger signs appear after operating for years [17]. In this situation, surface-mounted strain sensors are usually adopted. The surface-mounted FBG strain sensor mainly contains three types: bare FBG sensor, bare FBG with packaging substrate, and reusable FBG strain sensor. The first two types are attached to the surface of measured object using adhesive or by welding, such as the commercial sensors of "os 3100 " produced by Micron Optics (www. micronoptics.com) and "Smart patch" produced by Smart Fibres (www. smartfibres.com). Bare FBGs are liable to breakage and the strain transfer ratio which is an important influencing factor to measuring sensitivity can't be evaluated in laboratory once they are attached in 
project site [18]. Both bare FBG and FBG with packaging substrate have the drawback of no reusability which leads to higher costs and inconvenient replacement. In this context, the reusable surface-mounted FBG strain sensors have drawn much attention from various research communities in the past few years.

Several types of FBG sensor housing has been developed for different applications [19]-[23]. By using adhesive or glass solder, FBG is encapsulated or two-dot fixed on a substrate which is usually made of alloy steel or composite. Mounting holes are set at the end of elastic structure, through which the strain sensor could be fixed to the measured object via bolts. However, strain sensors of this kind lack sealing schedules, so the FBG and adhesive are exposed in air, which is liable to moisture and ultraviolet attack. Another kind of reusable FBG strain sensor with a major structure of cylinder [16], which is similar to the well-packaged embeddable strain sensor, is also reported. The ends of the sensor are processed into screw thread or fixed groove, which enable surface mounting to measured object via optional brackets with bolts, weld or grout. For example, commercial sensors of "os3610" produced by Micron Optics and "FS62-Surface Strain Sensor" produced by Fiber Sensing ( $w w w$.fibersensing.com).

When the reusable FBG strain sensors being installed, corresponding holes have to be drilled in the measured object too. Because the axial stiffness of the cylindrical structure is large, large external tension is required to make the sensor produce strain, which demands a stronger installation than the sensor itself. Otherwise, the install foundation will "absorb" some strain of the measured object, which could lead to an inaccurate measuring result. What's more, the number of mounting holes of the existing reusable FBG strain sensors (both the substrate structure and cylinder structure) is usually four or more, which mean that at least four corresponding holes have to be drilled in the measured object. On this occasion, the structural characteristics of the measured object are vulnerable to disruption, especially when the measured object is concrete.

In our previous work [24], [25], a reusable surface mounted FBG strain sensor has been developed and actually inspected in a highway bridge. The main body of the sensor is cylindrical structure with threaded ends. As mentioned above, the axial stiffness of the cylindrical sensor structure is so large, which results in that a $2 \mathrm{kN}$ force makes the sensor just produce $150 \mu \varepsilon$. Although an enhanced sensitivity structure has been adopted during sensor design and a strong installation method has been taken, the measured strain values of the highway bridge are much smaller the actual deformation. Eight holes were drilled in the bottom of the highway bridge to hold two brackets of just one sensor, which may bring bad influence to the structural integrity of the bridge.

In view of the exposed problems of our previous design and the other existing sensors, this paper introduces our recent work on the design and investigation of a new reusable surface mounted FBG strain sensor. The seal ability, number of mounting holes, and axial stiffness of the sensor have been considered and optimized. The mechanical structure design and measuring principle have been presented

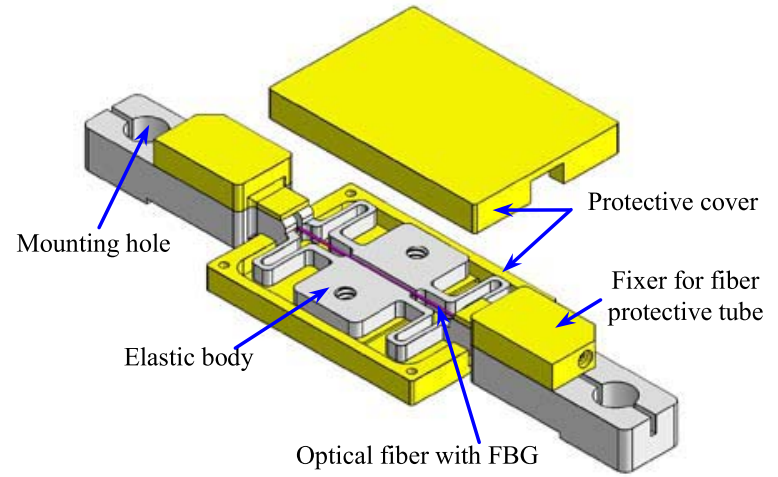

Fig. 1. Structure of the FBG strain sensor.

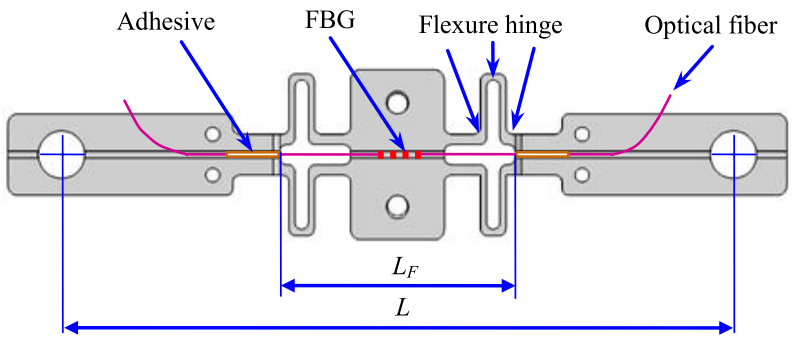

Fig. 2. Schematic of the elastic body and FBG arrangement.

then sensor prototype has been manufactured and full-scale tested.

\section{SENSOR DESIGN}

\section{A. Mechanical Structure and Measuring Principle}

Fig. 1 shows the mechanical structure design of the proposed FBG strain sensor. The sensor mainly includes an elastic body, a fiber with FBG, two protective covers, and two fixers for fiber protective tube. Two through-holes set at the ends of the elastic body are used as the mounting holes. So only two holes are needed to drill in the measured object, and then the sensor could be fixed by two screws and removed conveniently when the monitoring is finished. The lower protective cover is fixed through two screwed holes on the elastic body and the upper protective cover is fixed on the lower one by four mini bolts. The gap between the grooves of two protective covers and elastic body is filled with adhesive made of silicone rubber to seal the space in the covers where FBG is packaged. The stiffness of the elastic body will not be affected by the covers because the silicone rubber is soft. As shown in Fig. 2, flexure hinges have been adopt in the design of the key elastic body as their notable advantages such as compactness, no friction losses, no backlash and satisfactory stiffness. By using a commercial adhesive, a single mode optical fiber with FBG is adhered on the groove in the elastic body after an axial pretension, which allows the ability to measure negative strain.

In engineering practice, especially in the structural health monitoring of civil engineering, the deformation of concrete is usually small, so a strain sensor don't need a large measuring range but a high sensitivity. As illustrated in Fig. 2, an enhanced sensitivity design has been applied for the elastic body. The length between the two fixed points of the 


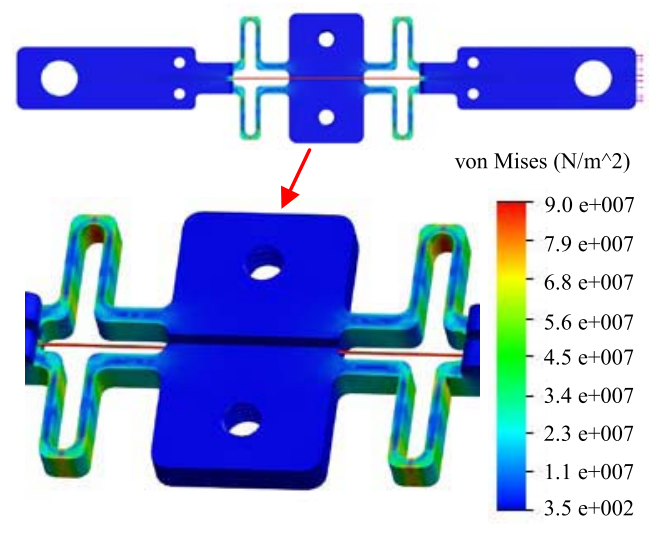

Fig. 3. Strain distributions on the elastic body simulated by FEA.

fiber is $L_{F}$, and the length between the two mounting holes is $L$ which is the gauge length of the sensor. Assuming that the deformation of the measured object between the mounting holes is $\Delta L$, the strain $\varepsilon$ is just $\Delta L / L$. The deformation of the area between the two fixed points of the fiber, in which the flexure hinges are contained, is $\Delta L_{F}$, so the strain of FBG $\varepsilon_{F B G}$ is $\Delta L_{F} / L_{F}$.

Strain distribution analysis of the elastic body and FBG under force loading has been simulated with the Finite Element Analysis (FEA) software of Solidworks simulation. The material of the elastic body is alloy steel, and parameters used in the simulation are as follows: Young's modulus $E_{1}=2.07 \times 10^{11} \mathrm{~Pa}$, Poisson's ratio $\mu_{1}=0.27$. And these parameters of the FBG made of glass are: Young's modulus $E_{2}=6.9 \times 10^{10} \mathrm{~Pa}$, Poisson's ratio $\mu_{2}=0.25$. Under a force of $20 \mathrm{~N}$, the simulated equivalent strain distribution has been presented in Fig. 3. The region between the two fixed points of FBG which contains the flexure hinges produce a clear strain and a deformation of $0.222 \mathrm{~mm}$, while the other region of the sensor almost has no strain and just a deformation of $0.00002 \mathrm{~mm}$. That means the flexure hinges with FBG almost take all the deformation of the measured area which length is $\mathrm{L}$ (the gauge length of the sensor), therefore $\triangle L_{F}$ equals to $\Delta L$. So, the ratio $K$ of strain values for $\varepsilon_{F B G}$ and $\varepsilon$ can be calculated as:

$$
K=\varepsilon_{F B G} / \varepsilon=\frac{\Delta L_{F} / L_{F}}{\Delta L / L}=\frac{L}{L_{F}}
$$

Because $L$ is large than $L_{F}$, the ratio is bigger than 1 . This is just the basic principle of the sensitivity enhancement design, and the enhanced coefficient $K$ can be adjusted by $L$ or $L_{F}$.

The center-reflecting wavelength shift of FBG $(\Delta \lambda)$ is influenced both by strain and temperature. The wavelength variation response to axial strain $(\Delta \varepsilon)$ and temperature change $(\Delta T)$ is given by:

$$
\frac{\Delta \lambda}{\lambda}=\left(\alpha_{f}+\xi\right) \Delta T+\left(1-P_{e}\right) \Delta \varepsilon
$$

Where $\lambda$ is the initial wavelength of FBG, $\alpha_{f}$ is the thermal expansion coefficient, $\xi$ is the thermal-optic coefficient and $P_{e}(\approx 0.22$ at room temperature $)$ is the effective photo-elastic coefficient. By combining (1) and (2), the strain $\varepsilon$ of the measured object can be obtained.

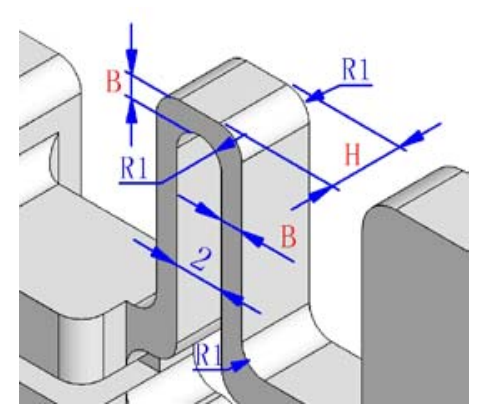

Fig. 4. Key dimension parameters of the flexure hinges.

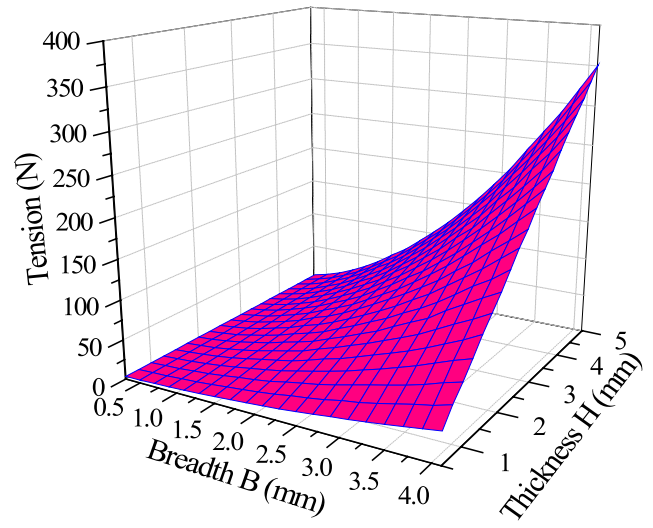

Fig. 5. The relation between thickness $\mathrm{H}$, breadth $\mathrm{B}$ and the required tension.

\section{B. Parameters Optimization for External Tension}

As we have discussed before, if the stiffness of the elastic body is too large, large external tension is required and the install foundation will "absorb" some strain of the measured object, which leads to an inaccurate measuring result. From the simulated strain distribution in Fig. 3, the deformation of the sensor is mainly produced by the flexure hinges. Therefore, the external tension depends on the stiffness of the flexure hinges. When the material is selected, the thickness $\mathrm{H}$ and breadth $\mathrm{B}$ of the flexure hinges are the key parameters which decide the stiffness.

As illustrated in Fig. 4, the thickness $H$, breadth $B$ and other parameters are labeled. Further FEA has been carried out to investigate the relationship between the external tension and the parameters of $H$ and $B$. We set the strain values of FBG $\varepsilon_{F B G}=1000 \mu \varepsilon$ to analyze the required tension under different $H$ and $B$. And then, consider the value of $H$ from 0.5 to $5 \mathrm{~mm}$ with a step of $0.25 \mathrm{~mm}, B$ from 0.3 to $4.1 \mathrm{~mm}$ with a step of $0.2 \mathrm{~mm}$. The relation between thickness $H$, breadth $B$ and the required tension has been presented in Fig. 5 (all the intersections and end-points of blue lines are FEA values).

In order to show the relation more intuitive, Fig. 6(a) presents the tension response versus $B$ variation with different $H$, which appears to be an exponential curve, whereas Fig. 6(b) presents the tension response versus $H$ variation with different $B$, which shows linear relationship. From the simulation results, it's obvious that the breadth $B$ of the flexure hinge plays a greater influence to the stiffness of the sensor structure than the thickness $H$. The corresponding dimension values could be selected according to the required tension for 

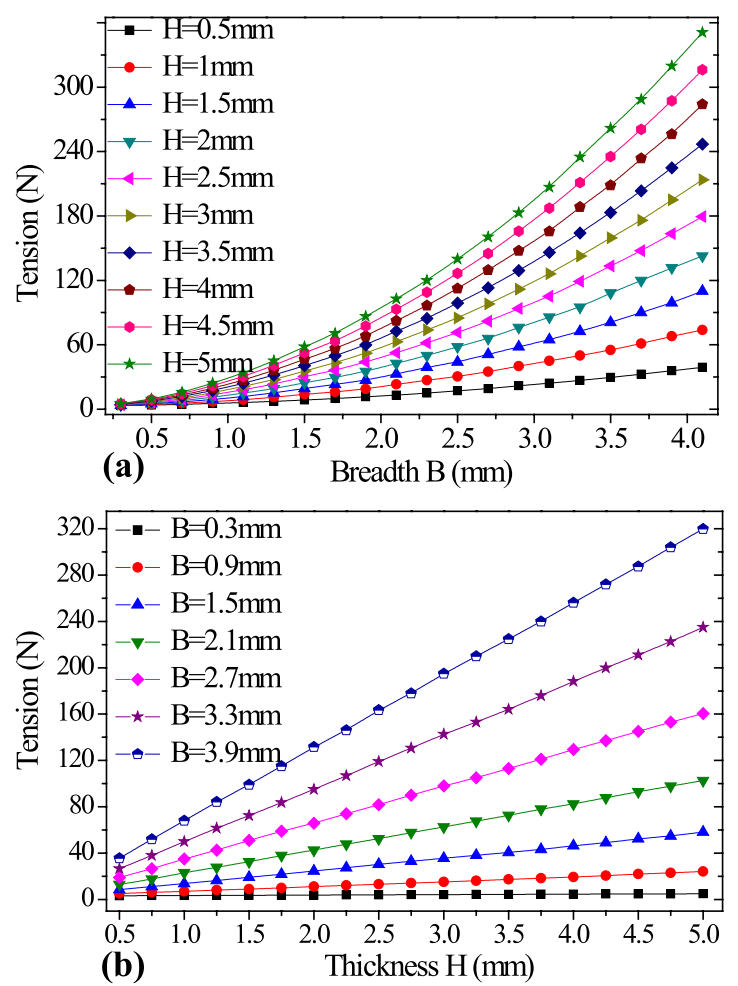

Fig. 6. FEA results. (a) The relation between $\mathrm{B}$ and tension with different $\mathrm{H}$. (b) The relation between $\mathrm{H}$ and tension with different $\mathrm{B}$.

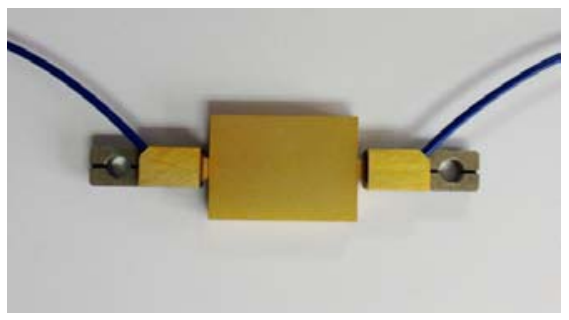

Fig. 7. Photo of the FBG strain sensor.

the target strain value of FBG. According to the FEA results and engineering practice, the parameters have been chosen as $H=3 \mathrm{~mm}$ and $B=1.5 \mathrm{~mm}$, which corresponds a tension about $34.65 \mathrm{~N}$.

\section{Sensor Prototype Manufacturing And EXPERIMENTS}

Besides the values of $H$ and $B$, the key parameters of $L$ and $L_{F}$ have been designed as $L=100 \mathrm{~mm}, L_{F}=$ $35 \mathrm{~mm}$, so the theoretical value of enhanced coefficient $K=100 / 35 \approx 2.857$. A FBG with reflectivity of $90 \%$ and central wavelength of $1535.002 \mathrm{~nm}$ has been adhered on the elastic body after an axial pre-tension of about $2.352 \mathrm{~nm}$. After assembling, a sensor prototype with a central wavelength of $1537.354 \mathrm{~nm}$ has been manufactured as shown in Fig. 7.

Fig. 8(a) shows the principle of experimental setup for calibration test. A commercial guide rail-slider system has been used to offer a kinematic pair with free sliding. The left mounting hole of sensor prototype and the guide rail have been fixed together on the experimental platform, whereas the right mounting hole has been fixed on the slider which (a)
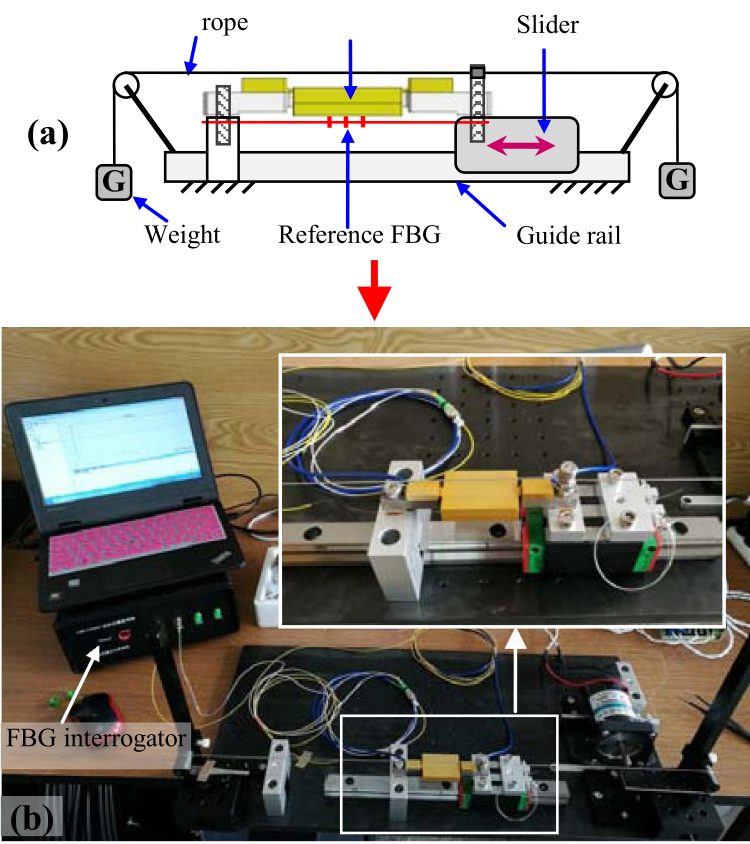

Fig. 8. Calibration test. (a) Principle of experimental setup. (b) Photo of the experimental setup.

could freely move along the guide rail. A reference FBG with an initial wavelength of $1535.306 \mathrm{~nm}$ has been fixed on the surface near the two mounting holes after an axial pre-tension of about $2.698 \mathrm{~nm}$. The length, reflectivity and bandwidth of the grating for the two FBGs are $5 \mathrm{~mm}, 90 \%$, and $0.2 \mathrm{~nm}$, respectively. Because the length between the two fixed points of the reference FBG is the same as the gauge length of the sensor, the strain detected by the reference FBG is just the strain of the measured object. Gravity of several standard weights $(500 \mathrm{~g})$ is used as the force load which acts on the right mounting hole through a wire rope. Here the acceleration of gravity in our laboratory $\left(30^{\circ} \mathrm{N}, 114^{\circ} \mathrm{E}\right)$ equals $9.793 \mathrm{~m} / \mathrm{s}^{2}$. A homemade FBG interrogator (acquisition frequency: $0 \sim 5000 \mathrm{~Hz}$, accuracy: $3 \mathrm{pm}$, resolution: $0.1 \mathrm{pm}$ ) is used to record the wavelength of the sensor and the reference FBG. Fig. 8(b) shows the photo of the experimental setup.

The calibration test begins by loading force on the sensor with a series of standard weights from $-58.758 \mathrm{~N}$ (towards left) to $58.758 \mathrm{~N}$ (towards right) with a step of $9.793 \mathrm{~N}$ (the gravity of two weights of $500 \mathrm{~g}$ ), then unloading it to $-58.758 \mathrm{~N}$ with the same step. This loading and unloading test process has been repeated four times at a stable room temperature. The time-history data of the four cycling tests has been plotted in Fig. 9.

Fig. 10 shows the eight curves of the relationship between wavelength shifts and force load obtained from the timehistory data. The variation patterns are linear and the repeatability error and hysteresis error for four tests of the strain sensor are $2.73 \%$ and $4.67 \%$, respectively. Fig. 11(a) shows variation of the average data of the four repeated tests and the linear fitting curves which indicate good fits. Turning the wavelength shift of the reference FBG to microstrain $(\mu \varepsilon)$ using (2) $(\Delta T=0)$, the wavelength shift of the strain sensor versus strain is shown in Fig. 11(b). From the fitted 


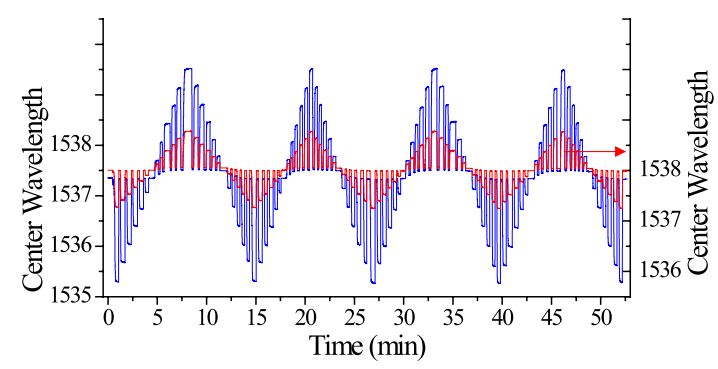

Fig. 9. Time-history curve of the four cycling tests.

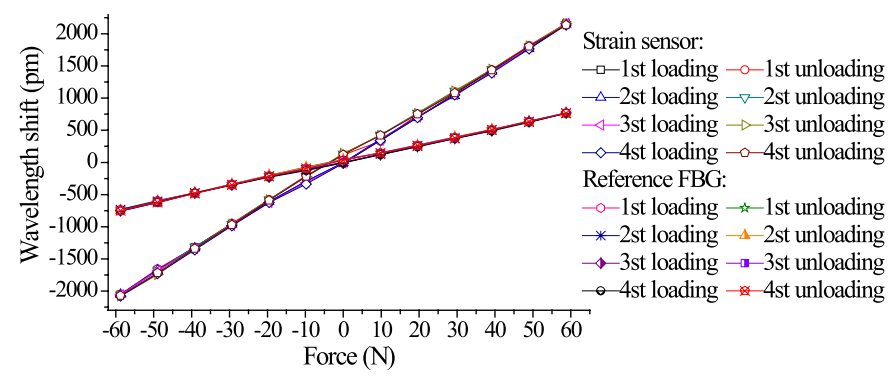

Fig. 10. Wavelength shifts versus force of the strain sensor and the reference FBG.
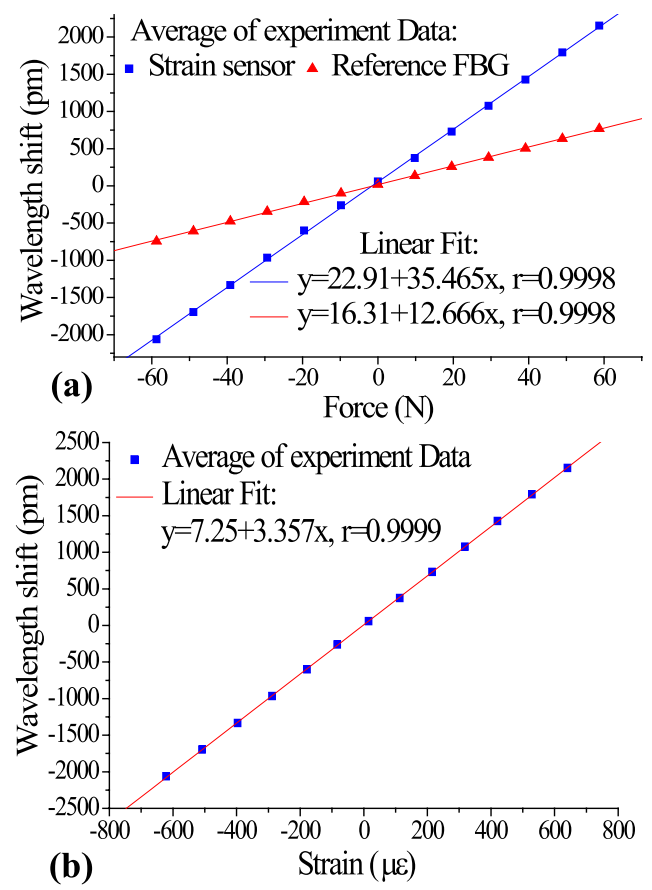

Fig. 11. (a) Linear fit of average of experiment data. (b) Average of wavelength shift versus strain and linear fit curve.

curve in Fig. 11(b), the sensitivity of the strain sensor is observed to be $3.357 \mathrm{pm} / \mu \varepsilon$ with a linearity of 0.9999 , and the measuring range is $-600 \sim 600 \mu \varepsilon$. When the FBG (central wavelength $=1537.354 \mathrm{~nm}$ ) of the strain sensor produces a strain of $1000 \mu \varepsilon$ as simulated before, corresponding wavelength shift could be calculated by $1537354 *(1-0.22) * 1000 * 10^{-6} \approx 1199 \mathrm{pm}$. From the fitted curve $(\mathrm{y}=22.91+35.465 \mathrm{x}, \mathrm{r}=0.9998)$ in Fig. 11(a), corresponding force of $33.16 \mathrm{~N}$ could be obtained, which is basically consistent with the simulation result of $34.65 \mathrm{~N}$. The enhanced coefficient $K$ could be obtained by the two fitted

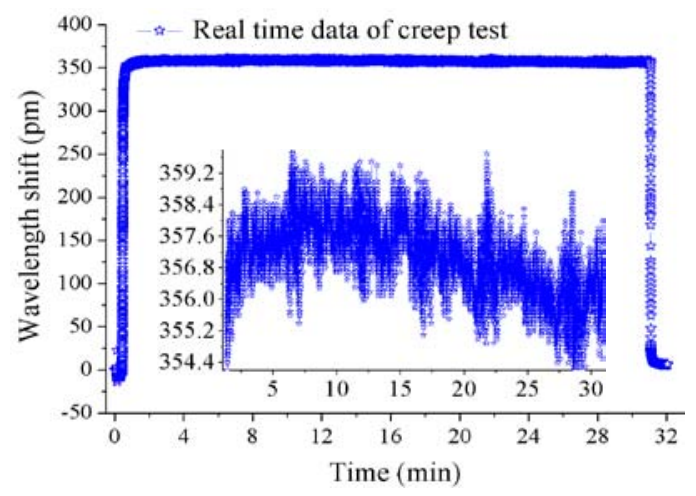

Fig. 12. Result of creep performance test.
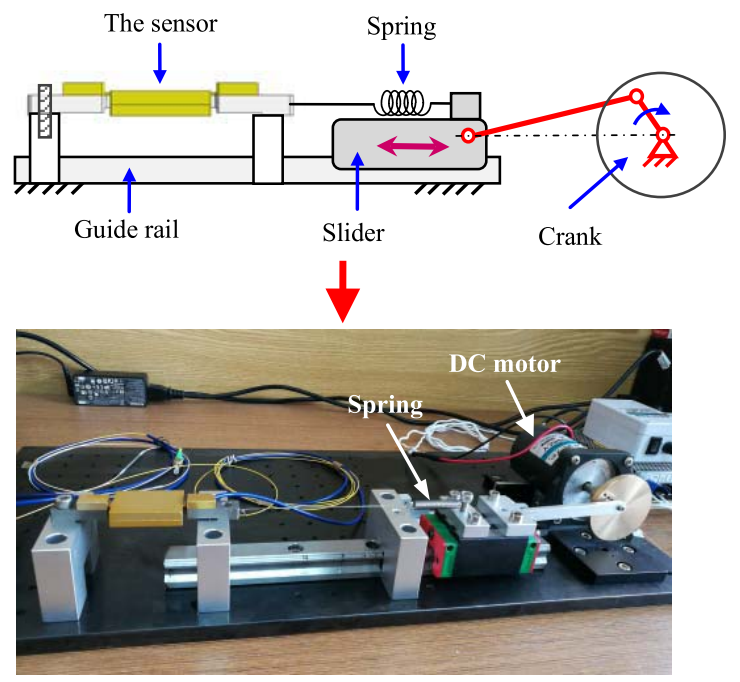

Fig. 13. Principle and photo of the experimental equipment for alternating strain test.

curves in Fig. 11 (a) as 35.465/12.666 $\approx 2.8$, or the ratio of the sensitivity of the strain sensor $(3.357 \mathrm{pm} / \mu \varepsilon)$ to the theoretical sensitivity $(1.199 \mathrm{pm} / \mu \varepsilon)$ of FBG with central wavelength near $1537 \mathrm{~nm}$ as $3.357 / 1.199 \approx 2.7998$, which are also consistent with the theoretical design value of 2.857 .

For long term measurement, the creep behavior is needed to know for real-world applications. During this test, a constant load of $9.793 \mathrm{~N}$ has been applied on the sensor over 30 minutes, and the wavelength shift is shown in Fig. 12. As we can see, the fluctuating value is within $5 \mathrm{pm}$, which demonstrates that the sensor has good creep resistance.

In engineering applications, for example, the deformation monitoring of bridge, frequent alternating strain brought by external load(cars, wind, etc ) often acts on the sensor in long time, which demands the sensor to have a applicability for this working condition. For example, in long-term use, if the material performance of the elastic structure and the soundness of the bond of FBG are affected by the frequent alternating strain, the pre-tension of FBG for negative strain detection will decrease, then the measuring range will change and can't reach $-600 \mu \varepsilon$, what's more, the sensitivity value may also change.

Special experimental equipment with the function of reciprocating motion has been designed using a crank-slider mechanism as shown in Fig. 13. The crank is powered by a DC motor 
TABLE I

ChANGES OF SENSITIVITY AND RANGE Under Alternating Strain Test

\begin{tabular}{ccc}
\hline \hline \multirow{2}{*}{ Number of Cycles } & Sensitivity $(\mathrm{pm} / \mu \varepsilon)$ & $\operatorname{Range}(\mu \varepsilon)$ \\
\hline 0 & 3.357 & $-600 \sim 600$ \\
$10^{3}$ & 3.21 & $-600 \sim 600$ \\
$10^{4}$ & 3.325 & $-600 \sim 600$ \\
$10^{5}$ & 3.41 & $-600 \sim 600$ \\
$10^{6}$ & 3.37 & $-600 \sim 600$ \\
\hline \hline
\end{tabular}
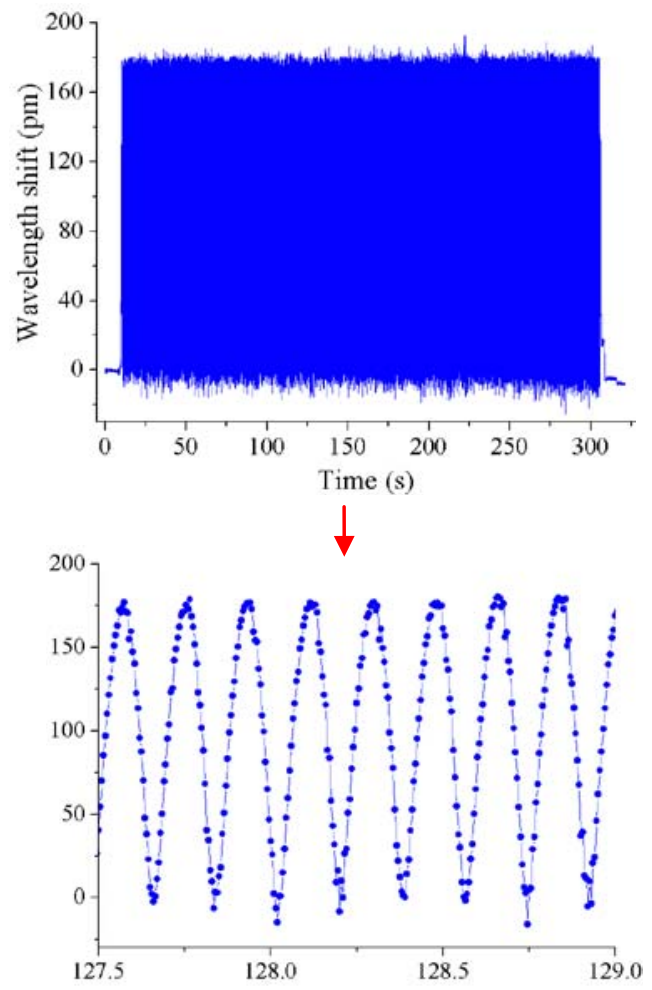

Fig. 14. Part of the experimental data for alternating strain test.

and a spring is used to connect the right mounting hole and the slider. Continuous alternating force has been applied on the sensor and the sensitivity and the measuring range of the sensor have been tested at different cycle numbers. Fig. 14 shows part of the experimental data recorded by FBG interrogator with a frequency of $200 \mathrm{~Hz}$, from which we can see that the amplitude and frequency of the alternating strain are $175 \mathrm{pm}$ and $5.5 \mathrm{~Hz}$, respectively. Table. 1 shows the changes of sensitivity and range at different cycle numbers. The sensitivity values have no abnormal change and the measuring ranges remain the same, which demonstrate that the alternating strain does not degrade the measurement capability.

In addition, because the wavelength shift of FBG is influenced by both axial strain and temperature, a temperaturecompensated sensor is needed for the proposed strain sensor to remove the wavelength shift brought by temperature variations. Therefore, the temperature sensitivity of the strain sensor has been tested for future use in practice. During the test, a thermostat is used to change the surrounding temperature of the strain sensor. The temperature is changed between $35{ }^{\circ} \mathrm{C}$

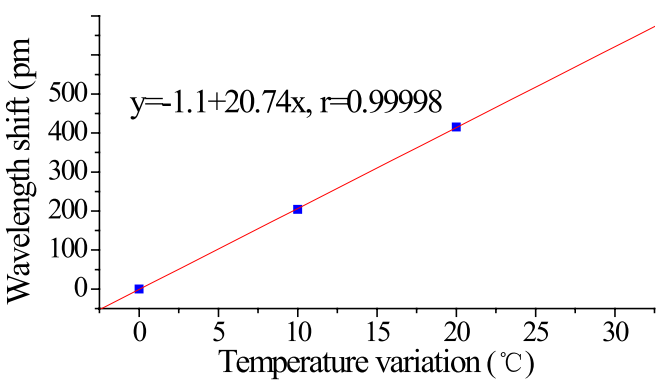

Fig. 15. Temperature sensitivity test result of the sensor.

and $65{ }^{\circ} \mathrm{C}$ and the sampling interval is $10{ }^{\circ} \mathrm{C}$. The wavelength shifts at each temperature point are recorded and linear fitted as shown in Fig. 15, which indicates a temperature sensitivity of $20.74 \mathrm{pm} /{ }^{\circ} \mathrm{C}$.

\section{CONCLUSION}

The work presented in this paper has described the design and test of an FBG-based reusable surface-mounted strain sensor. The content is presented in the following sequence: structure design, parameters optimization, sensor prototype manufacturing, and calibration test. Flexure hinges and sensitization structure have been adopted in elastic body, and then parameters optimization for external tension has been carried out. Sensor prototype with a central wavelength of $1537.354 \mathrm{~nm}$ has been manufactured.

Full performance tests have been carried out in strain response, creep, alternating strain, and temperature response. Experimental results show that the sensor has a high sensitivity of $3.357 \mathrm{pm} / \mu \varepsilon$ over a measuring range of $\pm 600 \mu \varepsilon$, a repeatability error of $2.73 \%$, and a hysteresis error of $4.67 \%$. The creep and alternating strain tests demonstrate good creep resistance and excellent measuring performance. Compared to the reported FBG strain sensors, the proposed strain sensor promises many potential applications in structural health monitoring, especially in the situation demands for replace ability and alternating strain measuring capability.

\section{REFERENCES}

[1] C. Pang et al., "A flexible and highly sensitive strain-gauge sensor using reversible interlocking of nanofibres," Nature Mater., vol. 11, no. 9, pp. 795-801, 2012.

[2] H. S. Park, H. Y. Lee, S. W. Choi, and Y. Kim, "A practical monitoring system for the structural safety of mega-trusses using wireless vibrating wire strain gauges," Sensors, vol. 13, no. 12, pp. 17346-17361, 2013.

[3] A. Ferreira da Silva, A. F. Goncalves, L. A. de Almeida Ferreira, F. M. M. Araujo, P. M. Mendes, and J. H. Correia, "A smart skin PVC foil based on FBG sensors for monitoring strain and temperature," IEEE Trans. Indust. Electron., vol. 58, no. 7, pp. 2728-2735, Jul. 2011.

[4] F. Marignetti et al., "Fiber Bragg grating sensor for electric field measurement in the end windings of high-voltage electric machines," IEEE Trans. Indust. Electron., vol. 63, no. 5, pp. 2769-2802, May 2016.

[5] D. Jia, Y. Zhang, Z. Chen, H. Zhang, T. Liu, and Y. Zhang, "A selfhealing passive fiber Bragg grating sensor network," J. Lightw. Technol., vol. 33, no. 10, pp. 2062-2067, May 2015.

[6] S. Javdani, M. Fabian, J. S. Carlton, T. Sun, and K. T. V. Grattan, "Underwater free-vibration analysis of full-scale marine propeller using a fiber Bragg grating-based sensor system," IEEE Sensors J., vol. 16 , no. 4, pp. 946-953, Feb. 2016.

[7] J. Chen, D. Huang, H. Zhao, Q. Wang, Y. Qiu, and D. Duan, "Fiber Bragg grating-based plane strain monitoring of aerostat envelope structures," Appl. Opt., vol. 52, no. 19, pp. 4631-4639, 2013. 
[8] Y. Guo, D. Zhang, J. Fu, S. Liu, S. Zhang, and F. Zhu, "Development and operation of a fiber Bragg grating based online monitoring strategy for slope deformation," Sensor Rev., vol. 35, no. 4, pp. 348-356, 2015.

[9] Y. Guo, D. Zhang, Z. Zhou, and F. Zhu, "Development and commissioning of FBG sensors for impact test of rock fall protective barrier," Sensor Rev., vol. 34, no. 4, pp. 343-348, 2014.

[10] S. Fan, L. Ren, and J. Chen, "Investigation of fiber Bragg grating strain sensor in dynamic tests of small-scale dam model," Struct. Control Health Monitor., vol. 22, no. 10, pp. 1282-1293, 2015.

[11] B. Korenko, M. Rothhardt, A. Hartung, and H. Bartelt, "Novel fiber-optic relative humidity sensor with thermal compensation," IEEE Sensors J., vol. 15, no. 10, pp. 5450-5454, Oct. 2015.

[12] Y. L. Park et al., "Real-time estimation of 3-D needle shape and deflection for MRI-guided interventions," IEEE/ASME Trans. Mechatronics, vol. 15, no. 6, pp. 906-915, Dec. 2010.

[13] G. Rajan et al., "Analysis of vibration measurements in a composite material using an embedded PM-PCF polarimetric sensor and an FBG sensor," IEEE Sensors J., vol. 12, no. 5, pp. 1365-1371, May 2012.

[14] F. Surre, T. Sun, and K. T. Grattan, "Fiber optic strain monitoring for long-term evaluation of a concrete footbridge under extended test conditionss," IEEE Sensors J., vol. 13, no. 3, pp. 1036-1043, Mar. 2013.

[15] P. Biswas et al., "Investigation on packages of fiber Bragg grating for use as embeddable strain sensor in concrete structure," Sens. Actuators A, Phys., vol. 157, no. 1, pp. 77-83, 2010.

[16] J. M. López-Higuera, L. Rodriguez-Cobo, A. Q. Incera, and A. Cobo, "Fiber optic sensors in structural health monitoring," J. Lightw. Technol., vol. 29, no. 4, pp. 587-608, Feb. 15, 2011

[17] R. H. Scott et al., "Commissioning and evaluation of a fiber-optic sensor system for bridge monitoring," IEEE Sensors J., vol. 13, no. 7, pp. 2555-2562, Jul. 2013.

[18] B. Torres, I. Payá-Zaforteza, P. A. Calderón, and J. M. Adam, "Analysis of the strain transfer in a new FBG sensor for structural health monitoring," Eng. Struct., vol. 33, no. 2, pp. 539-548, Feb. 2011.

[19] M. Majumder, T. K. Gangopadhyay, A. K. Chakraborty, K. Dasgupta, and D. K. B. Hattacharya, "Fibre Bragg gratings in structural health monitoring — Present status and applications," Sens. Actuators A, Phys., vol. 147, no. 1, pp. 150-164, 2008.

[20] Z. Yuan et al., "Structure optimization of fiber Bragg grating strain sensors," ACTA PHOTONICA SINICA, vol. 41, no. 11, pp. 1261-1266, 2012.

[21] W. Niu, Y. Ma, Y. Wu, Z. Yuan, Y. Gong, and Y. Rao, "Non-gel encapsulation process of a high temperature strain fiber Bragg grating sensor and its sensing properties," Chin. J. Sens. Actuators, vol. 26, no. 7, pp. 927-931, 2013 .

[22] T. Wang et al., "Fiber Bragg grating strain sensors for marine engineering," Photonic Sensors, vol. 3, no. 3, pp. 267-271, 2013.

[23] G. Ma, N. Mao, Y. Li, J. Jiang, H. Zhou, and C. Li, "The reusable load cell with protection applied for online monitoring of overhead transmission lines based based on fiber Bragg grating," Sensors, vol.16, no. 6, 2016, doi: 10.3390/s16060922.

[24] Y. Guo, D. Zhang, Z. Zhou, L. Li, and F. Zhu, "A novel surface-mounted FBG strain sensor and its application in highway bridge engineering," J. Opt. Laser, vol. 25, no. 3, pp. 435-441, 2014.

[25] L. Li, D. Zhang, H. Liu, Y. Guo, and F. Zhu, "Design of an enhanced sensitivity FBG strain sensor and application in highway bridge engineering," Photonic Sensors, vol. 4, no. 2, pp. 162-167, 2014.

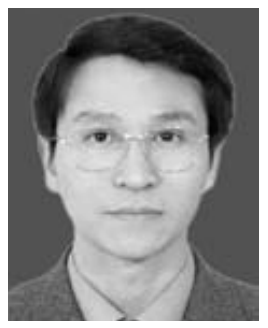

Jianyi Kong received the Ph.D. degree in mechanical engineering from Helmut Schmidt University, Germany, in 1994. He is currently a Professor and a Doctoral Supervisor of Mechatronic Engineering with the Wuhan University of Science and Technology, Wuhan, China. He has authored or co-authored 200 journal and conference publications, and six academic monographs. His research interests include controlled mechanism, intelligent systems, and advanced measuring technology.

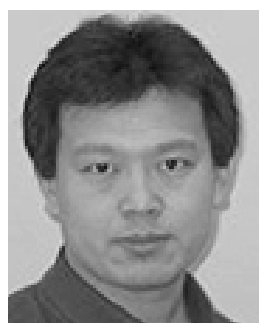

Honghai Liu (M'02-SM'06) received the Ph.D. degree in robotics from King's College London, London, U.K., in 2003. $\mathrm{He}$ held research appointments with the Universities of London and Aberdeen, and project leader appointments with large-scale industrial control and system integration industry. $\mathrm{He}$ is currently a Professor of Intelligent Systems with the University of Portsmouth, Portsmouth, U.K. His research interests include biomechatronics, pattern recognition, intelligent video analytics, intelligent robotics, robot sensing, and their practical applications with an emphasis on approaches that could make contribution to the intelligent connection of perception to action using contextual information.

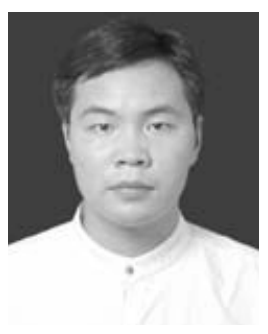

suppression
Dongtao Hu received the M.Eng. degree in mining engineering and the Ph.D. degree in safety technique and engineering from the Wuhan University of Technology, Wuhan, China, in 2007 and 2014, respectively. He is currently a Senior Engineer and Postdoctoral at the Wuhan University of Technology, Wuhan, China. He holds 16 issued Chinese patents. He has authored or co-authored seven journal and conference publications. His research interests include fiber Bragg grating sensing-based structural safety monitoring and industrial dust explosion

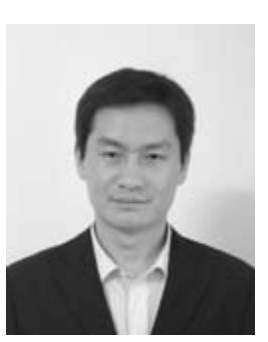

Yongxing Guo (M'16) received the M.Eng. and $\mathrm{Ph} . \mathrm{D}$. degrees in measurement control technology and instruments from the National Engineering Laboratory for Fiber Optic Sensing Technology, Wuhan University of Technology, Wuhan, China, in 2012 and 2014, respectively. He joined the Wuhan University of Science and Technology in 2014, where he is currently a Research Associate and a master's supervisor. He holds four issued Chinese patents. He has authored or co-authored 16 journal and conference publications. His current research interests include fiber Bragg grating sensing technology for mechanical equipment and civil engineering.

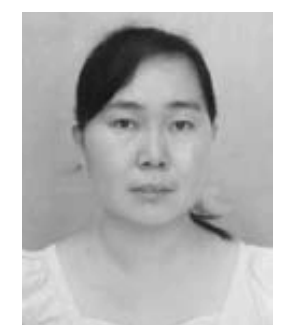

Li Qin received the Ph.D. degree in energy and power engineering from the Wuhan University of Technology, Wuhan, China, in 2014, respectively. She joined the Wuhan University of Science and Technology, as a Research Associate and a master's Supervisor in 2014. She holds three issued Chinese patents. She has authored or co-authored 12 journal and conference publications. Her current research interests include fiber Bragg grating-based advanced sensing technology. 